\title{
Mechanics of zero degree peel test on a tape - effects of large deformation, material nonlinearity, and finite bond length
}

\author{
Zezhou Liu ${ }^{\mathrm{a}}$, Helen Minsky b,1, Costantino Creton ${ }^{\mathrm{b}}$, Matteo Ciccotti ${ }^{\mathrm{b}}$, Chung-Yuen Hui ${ }^{\mathrm{a}, *}$

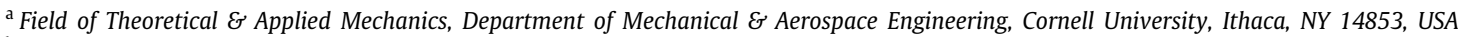 \\ ${ }^{\mathrm{b}}$ Laboratoire Sciences et Ingénierie de la Matière Molle, ESPCI Paris, PSL University, Sorbonne Université, CNRS, F-75005 Paris, France
}

\section{Introduction}

Many of us have experienced hanging a poster on a wall or a picture on a refrigerator with tape. Our intuition tells us that if the tape is too short, it is not going to hold. Interestingly, this mode of failure is rarely studied. Instead, most measurements on the mechanical strength of adhesive tape use a high angle peel test, e.g. the tape is peeled at an angle of 90 degrees. The mode of failure has been well studied [1-6]: the adhesive fails by nucleation and growth of cavities directly ahead of the peel front; the growth of these cavities creates tiny fibrils bridging the adhesive backing and the substrate [4-6] and failure occurs as these fibrils at the interface break. The same mode of failure can be observed in a probe tack test [7-13], where a flat cylindrical steel punch is brought into intimate contact with a thin adhesive film under a slight compressive force. This is followed by applying a tensile force to pull the punch off from the adhesive. In both tests, the adhesive is strongly confined, resulting in a very high triaxial state of stress which promotes cavitation $[13,14]$. This is not the

\footnotetext{
* Corresponding author.

E-mail address: ch45@cornell.edu (C.-Y. Hui).

1 Current address: Department of Physics and Astronomy, Carleton College, Northfield, MN 55057, USA.
}

case with the lap shear geometry, here our intuition suggests that the adhesive mainly deforms by shear. Hence, one may expect a different mode of failure. To the best of our knowledge, this mode of failure has not been studied in detail.

The lap shear geometry is a special case of a peel test where the peel force is aligned with the peel direction. In the literature, this is called the zero degree peel test. Here we remind the reader that the usual adhesive tape consists of a thin stiff backing $(\approx 20 \mu \mathrm{m}$ thick) bonded to an adhesive layer of similar thickness. The Young's modulus of the backing is on the order of several GPa [5], while the adhesive has a much lower modulus, in the $\mathrm{kPa}$ range. The mechanics of the peel test is well studied [1526]. Kendall's seminal work [3,27] determined the energy release rate in the steady peeling of an linearly elastic tape off a rigid substrate at arbitrary peel angles. More details on the complex state of stress at the peel front have been analyzed in detail by Thouless et al. [28] using a linear elastic fracture mechanics approach $[29,30]$. However, these works assume an infinitely thin adhesive layer, which is not the case for adhesive tapes. The key contribution in adhesive peeling is due to Kaelble [15] who accounts for the presence of a finite adhesive layer. The peel angle in Kaelble's theory is arbitrary, so the zero degree peel test is a special case of his analysis. 
Although Kaelble's analysis gives valuable insight into the mechanics of the zero degree peel test, it has several limitations. His analysis assumes that the adhesive is linearly elastic, that is, the strains are small and stress is directly proportional to the strain. This is certainly not the case for most common adhesives. Indeed, the shear strain in the adhesive layer can easily exceed $500 \%$ and $1000 \%$ strain is not uncommon [31-34]. In this regime, the small strain assumption breaks down. In addition, the assumption of material linearity also breaks down: the shear strain is no longer directly proportional to the shear stress. Indeed, most adhesives exhibit complex nonlinear stress-strain behavior. Roughly, for small strains, the stress-strain curve is linear; at larger strains, the adhesive softens, as indicated by a decrease in tangent modulus; finally, at high strains, the adhesive hardens [35]. In this work, we use a nonlinear material model which captures this feature. Yet another limitation involves local stress concentration: the complex 3D state of stress at the peel front cannot be well represented by a one-dimensional model. Finally, Kaelble assumes the bond length (the length of tape that is sticking to the substrate) is infinite [15], where the more interesting situation involves a finite bond length. Our analysis also introduces a feature absent from Kaelble's classical theory: we treat the peel front as an interface crack and determine the energy release rate of this crack. Our energy release rate expression is valid for arbitrary large deformation and for a wide class of hyperelastic material strain energy density functions.

The geometry considered in this work differs from the traditional lap joint test where the joint length is relatively short in comparison with relevant dimensions. Most lap joint analyses assume an adhesive layer of zero thickness $[29,36,37]$. In some studies, the adhesive layer is modeled as a cohesive zone $[29,37]$. Here we explicitly account for the finite thickness of the adhesive layer and we focus on the effect of large deformation and material nonlinearity of this layer.

In a previous work [38] we studied the large deformation mechanics of an adhesive tape subjected to zero-degree peel. In that study we focus on the situation where the bond length is infinite. Here we consider the effect of finite bond length on the mechanics of zero degree peel. It turns out a finite bond length introduces many interesting effects which are absent from the case of an infinitely long tape. For example, we shall demonstrate that the stress state at the free edge of the bond tape is highly triaxial and can nucleate cavities.

The organization of the paper is as follows: in Section 2 we study the strain and stress distribution in the adhesive layer using a nonlinear shear-lag model. We also present an exact expression for the energy release rate which is valid for large deformation and nonlinear materials. We compare our results with the linear theory established by Kaelble [15]. Our analytic results are validated by a nonlinear FE model. In Section 3 we consider the stress and strain near the edges, where the shearlag model breaks down. Summary and discussion are given in Section 4.

\section{Theoretical analysis}

\subsection{Statement of problem and geometry}

The geometry is shown in Fig. 1(a). The bond length is denoted by $L$ and the initial thickness of the adhesive layer is denoted by $h$. The backing layer has thickness $h_{b}$ which is on the same order as $h$. In the following we assume that the backing layer is linear elastic, with plane strain Young's modulus $E_{b}^{*}$. As the width of the tape is much greater than its thickness, we assume plane strain deformation where the out-of-plane displacement $u_{3}$ is zero and the in-plane displacements $u_{\alpha}(\alpha=1,2)$ depend only on the in-plane coordinates $\left(x_{1}, x_{2}\right)$. Another assumption is that the hanging weight is uniformly distributed across its width, therefore the appropriate force measured in a plane strain analysis, denoted by $F$, is defined as the weight divided by the width of the tape and has units of force per unit length. This force $F$ is applied horizontally on one end of the tape (note $F$ is negative in our coordinate system). Since the substrate is rigid, it can be extended pass the origin in the negative $x_{1}$ direction, so the point $x_{1}=x_{2}=0$ can be considered as the tip of an interface crack (see Fig. 1(b)). After load is applied, the material point at $\left(x_{1}, x_{2}\right)$ moves to $\left(y_{1}, y_{2}\right)$ with respect to the same coordinate system, thus, $y_{\alpha}=x_{\alpha}+u_{\alpha}$.

\subsection{Special case of a linear elastic adhesive - small strains}

To position this work in relation to previous works and to motivate the idea, we first consider a situation with a linear elastic adhesive layer with shear modulus $\mu$; this assumption is valid provided that the deformation of the adhesive layer or the applied force $F$ is small. A straightforward calculation based on the shear-lag model $[15,39]$ shows that shear strain in the adhesive layer is (see the Supplementary Information (SI) for details)

$\gamma=\frac{F}{\mu l_{L T}} \frac{\cosh (\bar{L}-\eta)}{\sinh \bar{L}}$, where $\bar{L}=L / l_{L T}, \eta=x_{1} / l_{L T}$.

The tension in the backing layer $\tau_{11}^{B}$ and the shear stress in the adhesive layer $\tau_{12}$ are given by

$\tau_{11}^{B}=-\frac{E_{b}^{*} h}{\sinh \bar{L}} \frac{F}{\mu l_{L T}} \sinh (\bar{L}-\eta), \quad \eta \in[0, \bar{L}] \quad$ Backing layer,

$\tau_{12}=\frac{F}{l_{L T} \sinh \bar{L}} \cosh (\bar{L}-\eta), \quad \eta \in[0, \bar{L}], \quad$ Adhesive layer

respectively. Note in the shear-lag model, the backing layer is subjected to pure in-plane tension and because the strains are assumed to be small, the only non-vanishing stress in the adhesive layer is the shear stress $\tau_{12}$.

Equations (1a)-(1c) contain a very important length scale which is the load transfer length $l_{L T}$. It is

$l_{L T}=\sqrt{E_{b}^{*} h h_{b} / \mu}$.

This is the length of the adhesive tape that carries most of the load - more precisely, it is the characteristic decay distance of the shear stress along the tape. For a typical tape, the shear modulus of the adhesive is on the order of tens of $\mathrm{kPa}$, and the plane strain Young's modulus of the backing layer is on the order of GPa. The thicknesses of the adhesive and backing layers are on the order of tens of microns. Taking $h=h_{b}=20$ microns, $E_{b}^{*} \approx 3 \mathrm{GPa}$ and $\mu=10 \mathrm{kPa}$ [5], the load transfer length is about $10 \mathrm{~mm}$.

\subsection{Extension to large deformation and nonlinear stress-strain behavior}

The results in Section 2 are based on two simplifying assumptions: (1) the strains are small in the adhesive layer so we can avoid finite strain kinematics; (2) the adhesive is linearly elastic. Both assumptions are unrealistic in practical applications where the strains in the adhesive can reach $1000 \%$ [31-34]. Here we explore how removal of these two assumptions affects the results above. In our previous work [38], we assumed the bond length is infinite and modeled the nonlinear elastic behavior of the adhesive using a three-term Yeoh solid. We showed that the stress and deformation in the adhesive layer can be accurately predicted 


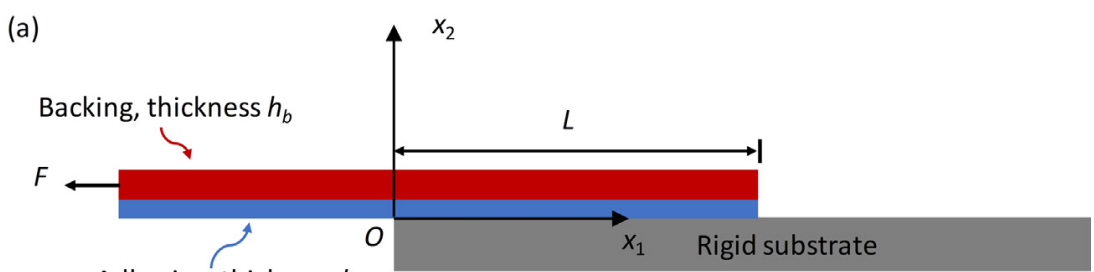

Adhesive, thickness $h$

(b)

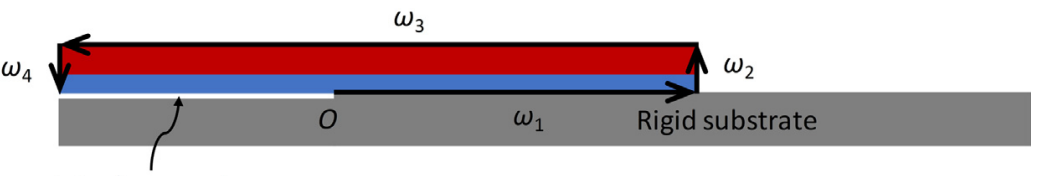

Interface crack

Fig. 1. Schematics of a zero degree peel test. (a) $\mathrm{L}$ is the finite bond length. The adhesive and backing thickness are denoted by $h$ and $h_{b}$, respectively. The applied force $\mathrm{F}$ is usually provided by a hanging weight per unit width. (b) The overhanging adhesive surface up to the peel front $\left(x_{1}<0, x_{2}=0\right)$ can be seen as an interface crack. The path $\omega=\omega_{1}+\omega_{2}+\omega_{3}+\omega_{4}$ used in the J integral is illustrated by black arrows (see Section 2.5).
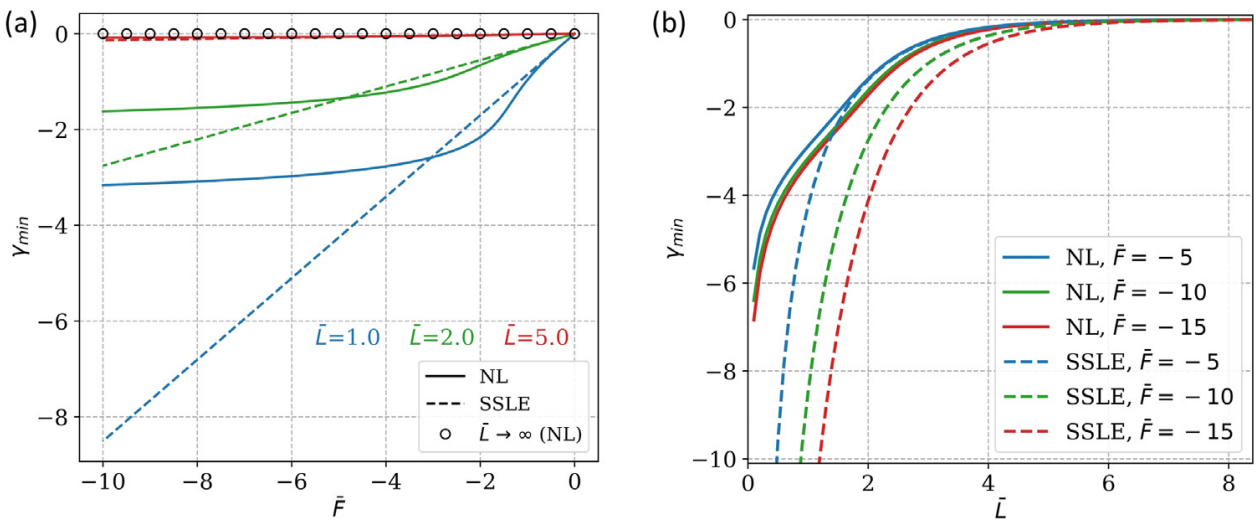

Fig. 2. (a) Minimum shear strain $\gamma_{\min }$ versus the normalized force $\bar{F}$ for three normalized bond lengths $\bar{L}$. The dashed and solid lines represent SSLE and NL solutions, respectively. The limiting case of $\bar{L}=\infty$ is plotted as circles. (b) Minimum shear strain $\gamma_{\min }$ versus the normalized bond lengths $\bar{L}$ for different normalized force $\bar{F}$.

by a nonlinear shear-lag model. Here we use the same nonlinear shear-lag model to study the finite bond length problem.

The average shear strain in the adhesive layer is $\gamma=u_{b} / h$, where $u_{b}$ is the horizontal displacement in the backing layer. Since the equation governing $\gamma$ was obtained in our previous work [38], we state it here without derivation, leaving some of the details in the SI. Briefly, Eq. (2) below expresses the equilibrium of a material element in the backing layer, that is, the net tension on this element must be balanced by the shear stress $\tau(\gamma)$ exerted by the adhesive layer to maintain equilibrium. Due to material nonlinearity, $\tau(\gamma)$ is a nonlinear function of the shear strain $\gamma$

$\frac{d^{2} \gamma}{d \eta^{2}}=\hat{\tau}(\gamma)$

where $\eta=x_{1} / l_{L T}$ is the normalized distance from the crack tip and $\hat{\tau}(\gamma) \equiv \tau(\gamma) / \mu$ is the normalized shear stress and $\mu$ is the small strain shear modulus in the Yeoh model. The strain energy density function $W$ of the Yeoh solid is

$W=C_{1}\left(I_{1}-3\right)+C_{2}\left(I_{1}-3\right)^{2}+C_{3}\left(I_{1}-3\right)^{3}$

where the $C_{k}$ 's are material parameters with units of stress, $I_{1}$ is the trace of the right Cauchy-Green tensor. For incompressible materials, $C_{1}=\mu / 2$. Softening is controlled by setting $C_{2}<0$ and strain hardening is controlled by $C_{3}>0$. In our previous work, we have shown that the normalized shear stress in Eq. (2) is related to the shear strain by

$\hat{\tau}=f(\gamma) \gamma, \quad f(\gamma)=\left(1+\frac{4 C_{2}}{\mu} \gamma^{2}+\frac{6 C_{3}}{\mu} \gamma^{4}\right)$. and

$I_{1}-3 \approx \gamma^{2}$

The dimensionless function $f(\gamma)$ in Eq. (3b) represents strain hardening behavior. For example, $C_{2}=C_{3}=0$ corresponds to a neo-Hookean solid. It is interesting to note the shear strain distribution is governed by the same equation whether or not the adhesive is linear elastic or neo-Hookean. However, it must be noted that the stress state in the adhesive is highly dependent on whether the material is linear or nonlinear, as revealed in Section 2.6. The boundary conditions are

$\left.\gamma\right|_{\eta=0}=\gamma_{0},\left.\frac{d \gamma}{d \eta}\right|_{\eta=L / l_{L T}}=0$.

Here $\gamma_{0}$ denotes the maximum shear strain which occurs at $x_{1}=$ 0 . The last condition in (4b) states that the tension in the backing layer at $x_{1}=L$ is zero. The maximum shear strain $\gamma_{0}$ is determined by the peel force $F$ which is the integral of the shear stress along the adhesive/backing interface, i.e.,

$F=\mu l_{L T} \int_{0}^{L / l_{L T}} f(\gamma) \gamma d \eta$.

The shear strain distribution $\gamma$ is obtained by solving equation (2) subjected to the boundary conditions $(4 a, b)$. It is (see the SI for details)

$\eta=-\int_{\gamma_{0}}^{\gamma} \frac{d w}{\sqrt{\left(w^{2}-\left(\gamma_{\min }\right)^{2}+\frac{c_{2}}{2}\left(w^{4}-\left(\gamma_{\min }\right)^{4}\right)+c_{3} \frac{w^{6}-\left(\gamma_{\min }\right)^{6}}{3}\right)}}$, 
where $\gamma_{\min }$ denote the minimum shear strain at $x_{1}=L$ or $\eta=$ $L / l_{L T}, w$ is a dummy integration variable, and

$c_{2}=4 C_{2} / \mu, \quad c_{3}=6 C_{3} / \mu$.

Eq. (5) motivates us to introduce a normalized peel force $\bar{F} \equiv$ $F / \mu l_{L T}$. The peel force can be evaluated exactly (see the SI); it is

$\bar{F}=\gamma_{\min } \sqrt{\rho^{2}-1+\frac{c_{2}}{2}\left(\gamma_{\min }\right)^{2}\left(\rho^{4}-1\right)+\frac{c_{3}}{3}\left(\gamma_{\min }\right)^{4}\left(\rho^{6}-1\right)}$,

where $\rho=\gamma_{0} / \gamma_{\min }$

Note that both $\gamma_{0}, \gamma_{\min }$ are unknowns which depend on the peel force and the bond length. To determine these relationships, we evaluate Eq. (6a) at $\eta=L / l_{L T}$; after some simple algebra, it reduces to the following equation relating bond length and the maximum and minimum shear strains, i.e.,

$\bar{L} \equiv \frac{L}{l_{L T}}$

$=\int_{1}^{\rho} \frac{d w}{\sqrt{w^{2}-1} \sqrt{1+\frac{c_{2}\left(\gamma_{\min }\right)^{2}}{2}\left(w^{2}+1\right)+\frac{c_{3}\left(\gamma_{\min }\right)^{4}}{3}\left(w^{4}+w^{2}+1\right)}}$.

Eqs. (7) and (8) give two equations to determine $\gamma_{0}$ and $\gamma_{\min }$ as a function of $\bar{F}=F / \mu l_{L T}$ and $\bar{L}=L / l_{L T}$. Specifically, these two equations imply that these two strains depend only on (for the sake of simplifying notations, we have not indicated their dependence on $c_{2}$ and $c_{3}$ )

$\gamma_{0}=f_{0}(\bar{F}, \bar{L}), \gamma_{\min }=f_{\min }(\bar{F}, \bar{L})$.

Unfortunately, there is no closed form solution for the integrals (6a) and (8) unless $c_{2}=c_{3}=0$. In the SI, we show how to numerically solve for $\gamma_{0}$ and $\gamma_{\min }$ as a function of $\bar{F}$ and $\bar{L}$ and to determine the shear strain distribution in the adhesive layer. For the linear elastic case, these strains are directly proportional to the peel force and from (1a), the maximum and minimum shear strains are:

$\gamma_{0}=\frac{F \operatorname{coth} \bar{L}}{\mu l_{L T}}, \gamma_{\min }=\frac{F}{\mu l_{L T} \sinh \bar{L}}, \rho=\frac{\gamma_{0}}{\gamma_{\min }}=\cosh \bar{L}$.

\subsection{Numerical results}

The minimum shear strain $\gamma_{\text {min }}$ is plotted against the normalized peel force $\bar{F}$ for different normalized bond lengths $\bar{L}$ in Fig. 2. The special case of $\bar{L}=\infty$ where it vanishes is shown as circles. Unless otherwise stated, we use the same parameters $c_{2}=-0.0948$ and $c_{3}=0.00996$ from our previous study [38] in all the figures below. The special case of small strain linear elasticity (SSLE) (solid lines) are also plotted as a comparison. In the linear theory, $\gamma_{\min }$ and $\gamma_{0}$ are directly proportional to $\bar{F}$ (see Eqs. (10a,b)). Fig. 2(a) shows that as long as $\bar{F}>-1$, there is no significant differences between linear and nonlinear theory. However, for larger forces, the linear theory overpredicts $\gamma_{\text {min }}$ considerably. The reason is that the nonlinear material strain hardens significantly; therefore, for large force, the nonlinear material deforms much less. Indeed, due to strain hardening, $\gamma_{\min }$ increases very slowly with the applied force for $\bar{F}<-5$. This result is also illustrated in Fig. 2(b), where $\gamma_{\text {min }}$ is plotted against normalized bond length for large normalized forces. In contrast to the linear theory, for large applied force, $\gamma_{\min }$ approximately depends only on the bond length in the high force regime; the curves of $\gamma_{\text {min }}$ versus $\bar{L}$ practically lies on top of each other. Mathematically, in this regime the minimum shear strain

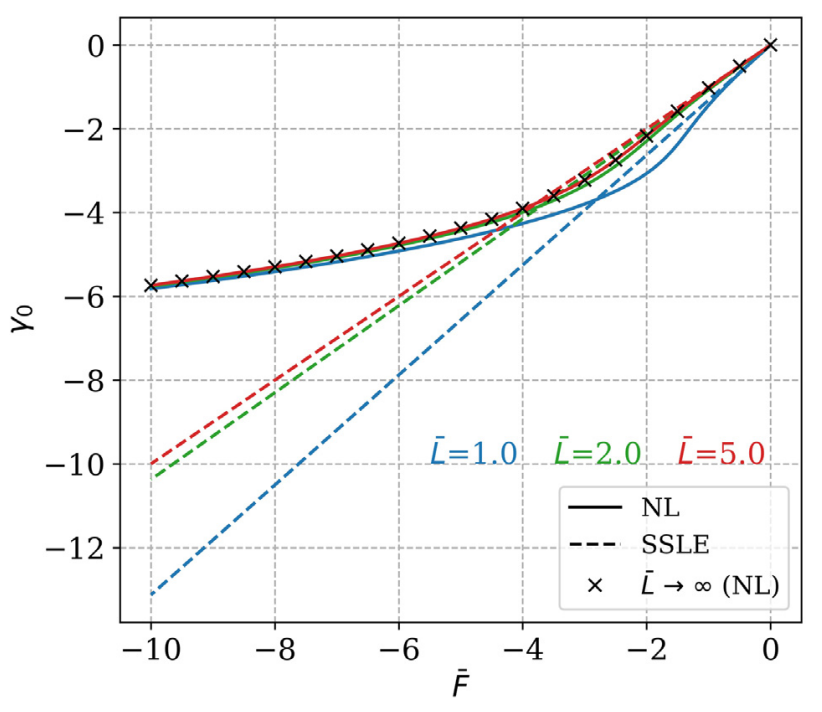

Fig. 3. Maximum shear strain $\gamma_{0}$ versus the normalized force $\bar{F}$ for three normalized bond lengths $\bar{L}$. The dashed and solid lines represent SSLE and NL solutions, respectively. The limiting case of $\bar{L}=\infty$ is plotted as cross symbols.

is approximately independent of the peel force, i.e.,

$\gamma_{\min } \approx f_{\min }(\bar{L}), \bar{F} \ll-1$

Fig. 3 plots the maximum shear strain $\gamma_{0}$ versus the normalized force $\bar{F}$ for three normalized bond lengths. We also plotted the prediction of the linear theory for comparison. Note in particular, the special case of $\bar{L}=\infty$ (cross symbols) sets a lower bound for the peel force (for the same $\gamma_{0}$ ). As expected, for small $\bar{F}$, there is no significant difference between the linear and nonlinear theory. The effect of strain softening occurs at peel forces roughly between -4 to -2 , after which strain hardening takes over and the increase in maximum shear stress is much lower than that predicted by the linear theory. An interesting result is that, $\gamma_{0}$ versus $\bar{F}$ curves for different normalized bond lengths lie on top of each other for large $\bar{F}$. Mathematically, this result is:

$\gamma_{0} \approx f_{0}(\bar{F}), \bar{F} \ll-1$

Thus, decreasing the bond length in the high force regime has only small effect on the maximum shear strain - a surprising result.

The shear strain distribution is plotted in Fig. 4(a) for normalized bond length $\bar{L}=1$ for three different normalized peel forces. The prediction of the linear theory is also plotted for comparison. In Fig. 4(b), these distributions are plotted for a fixed force $(\bar{F}=-10)$ with different curves representing different normalized bond lengths. In order to plot these curves in one figure, we use a normalized position $\bar{r}$, defined by $\bar{r} \equiv x_{1} / L=\eta / \bar{L}$, as the horizontal axis.

\subsection{Energy release rate}

The energy release rate $\Gamma$ of the interface crack in Fig. 1(b) can be computed using the path independent $J$ integral in the undeformed reference configuration [40,41], which is

$J=\int_{\omega} W d y-P_{\alpha \beta} N_{\beta} u_{\alpha, 1} d s, \omega=\omega_{1}+\omega_{2}+\omega_{3}+\omega_{4}, \alpha, \beta=1,2$,

where $P_{\alpha \beta}, N_{\beta}$ are the components of nominal stress tensor and unit normal in the reference configuration respectively. Also $u_{\alpha, 1}$ 

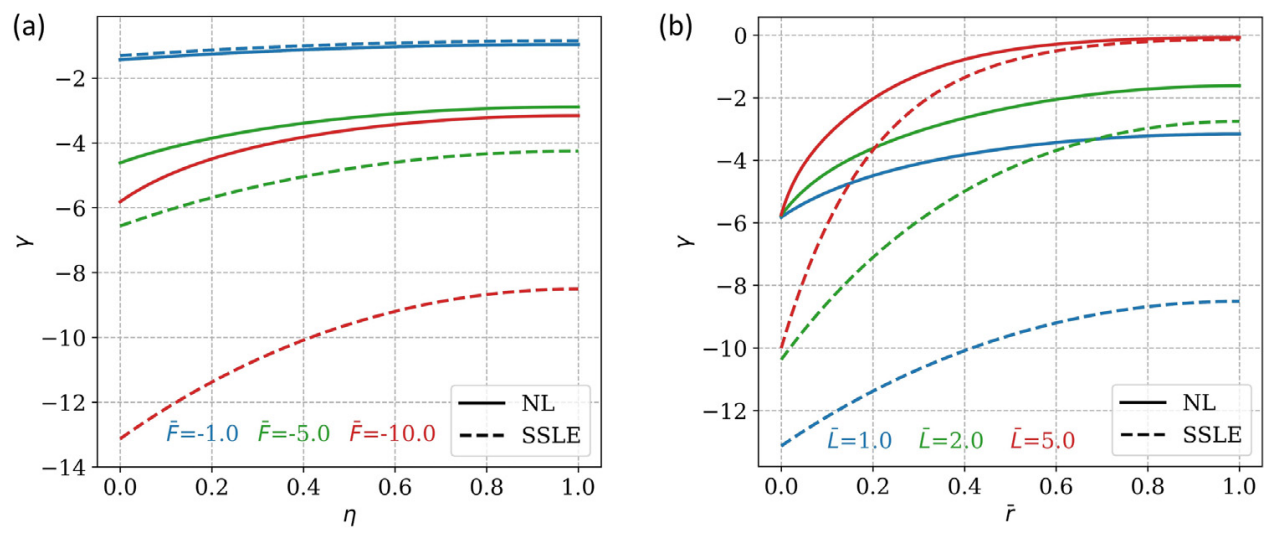

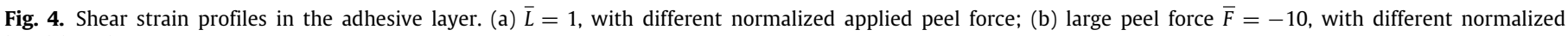
bond lengths.

is defined as $\partial u_{\alpha} / \partial x_{1}$. For the path $\omega$ chosen in Fig. 1(b), the integral is zero on $\omega_{1}$ and $\omega_{3}$. On $\omega_{2}$ the traction is zero, so $P_{\alpha \beta} N_{\beta} u_{\alpha, 1}$ is zero, thus only the first term contributes on $\omega_{2}$ and it is

$\int_{\omega_{2}} W d y=\int_{0}^{h_{\text {tape }}} W\left(x_{1}=L, x_{2}\right) d x_{2}$,

where $h_{\text {tape }}=h_{b}+h$. Note that $W$ cannot be evaluated exactly on this path, since the corners can have weak singularities. The integral on path $\omega_{4}$ can be readily evaluated, since the adhesive on this path is practically stress free and all the loads are carried by the much stiffer backing layer, which is linear elastic. Hence

$\int_{\omega_{4}} W d y-P_{\alpha \beta} N_{\beta} u_{\alpha, 1} d s=\frac{\left(F / h_{b}\right)^{2}}{2 E_{b}^{*}} h_{b}$.

Putting all this together, the energy release rate $\Gamma=J$ is

$\Gamma=\frac{F^{2}}{2 E_{b}^{*} h_{b}}+\int_{0}^{h_{b}+h} W\left(x_{1}=L, x_{2}\right) d x_{2}$

Physical consideration suggests that $W\left(x_{1}=L, x_{2}\right)$ must increase with decreasing $L$ since the same load has to be distributed over a smaller bond area. Since $\mathrm{d} L=-d c$ as the crack grows by $d c$, Eq. (16) implies that the energy release rate $\Gamma$ increases with crack growth for any finite bond length $L$. Note that the energy release rate given by Eq. (16) is valid for any nonlinear elastic solid and arbitrary large deformation of the adhesive layer.

\section{Special case of a linear elastic adhesive}

The special case of a linear elastic adhesive can be readily determined using Eq. (1a)-(1c). The strain energy density of the adhesive at $x_{1}=L$ can be estimated using the shear-lag model,

$W(x=L)=\left.\frac{1}{2} \tau_{12} \gamma\right|_{x=L}=\frac{1}{2 \mu}\left(\frac{F}{l_{L T} \sinh \left(L / l_{L T}\right)}\right)^{2}$.

The energy release rate equation (16) is

$$
\begin{aligned}
\Gamma & =\frac{F^{2}}{2 E_{b}^{*} h_{b}}+\frac{h}{2 \mu}\left(\frac{F}{l_{L T} \sinh \left(L / l_{L T}\right)}\right)^{2} \\
& =\frac{F^{2} \cot h^{2}\left(L / l_{L T}\right)}{2 E_{b}^{*} h_{b}}=\Gamma_{\infty} \cot h^{2}\left(L / l_{L T}\right) .
\end{aligned}
$$

The first term in Eq. (18) is independent of bond length, while the second term in (18) vanishes as $L$ goes to infinity, hence the first term, $\Gamma_{\infty} \equiv \frac{F^{2}}{2 E_{b}^{*} h_{b}}$ is the energy release rate for the case of infinite bond length. Fig. 5(a) plots the normalized energy release rate $\Gamma / \Gamma_{\infty}$ versus $\bar{L}$.

\section{Energy release rate for nonlinear adhesive and large deforma- tion}

We evaluate the energy release rate by Eq. (16) using the results in Section 2.3, specifically equations (3a) and (3c). The key is to compute the strain energy density $W$ in the adhesive layer at $x_{1}=L$. In the nonlinear shear-lag model, the energy density function $W$ at the bond end is related to the shear strain there by

$W\left(x_{1}=L\right)=\frac{\mu}{2} \gamma_{\min }^{2}\left[1+\frac{c_{2} \gamma_{\min }^{2}}{2}+\frac{c_{3} \gamma_{\min }^{4}}{3}\right]$.

Note that the strain energy density increases rapidly with the minimum shear strain $\gamma_{\text {min }}$ at $x_{1}=L$. The energy release rate based on Eqs. (16) and (19) is

$\Gamma=\frac{F^{2}}{2 E_{b}^{*} h_{b}}+\frac{\mu h}{2} \gamma_{\min }^{2}\left[1+\frac{c_{2} \gamma_{\min }^{2}}{2}+\frac{c_{3} \gamma_{\min }^{4}}{3}\right]$.

Note that if we set $c_{2}=c_{3}=0$ in Eq. (20), we recover the linear theory energy release rate given by Eq. (18). Here we emphasized that $\gamma_{\min }$ is a nonlinear function of $F$ and $L$, hence the energy release rate is no longer proportional to $F^{2}$. Consistent with our previous notation, we decompose the energy release rate into two parts,

$\Gamma=\Gamma_{\infty}+\Gamma_{L}, \quad \Gamma_{L} \equiv \frac{\mu h}{2} \gamma_{\min }^{2}\left[1+\frac{c_{2} \gamma_{\min }^{2}}{2}+\frac{c_{3} \gamma_{\min }^{4}}{3}\right]$.

The first term in (21), $\Gamma_{\infty}=F^{2} / 2 E_{b}^{*} h_{b}$ is independent of the bond length $L$ whereas the second term $\Gamma_{L}$ is very sensitive to it.

The analysis above provides several interesting results:

(1) The effect of adhesive modulus only comes in through the load transfer length; for long bond length, the energy release rate is insensitive to the adhesive thickness and its elastic properties.

(2) Griffith criterion states that crack growth occurs when

$\Gamma=G_{c}$,

where $G_{c}$ is the critical interfacial fracture energy which we assumed to be a material constant, independent of crack length. Eq. (20) tells us when the bond length is long in comparison with the load transfer length, the second term is small since $\gamma_{\min } \rightarrow 0$, hence

$\Gamma \approx \frac{F^{2}}{2 E_{b}^{*} h_{b}}$. 
(a)

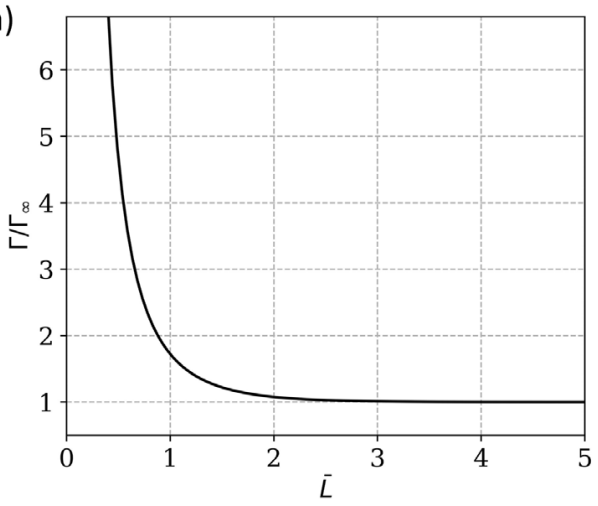

(b)

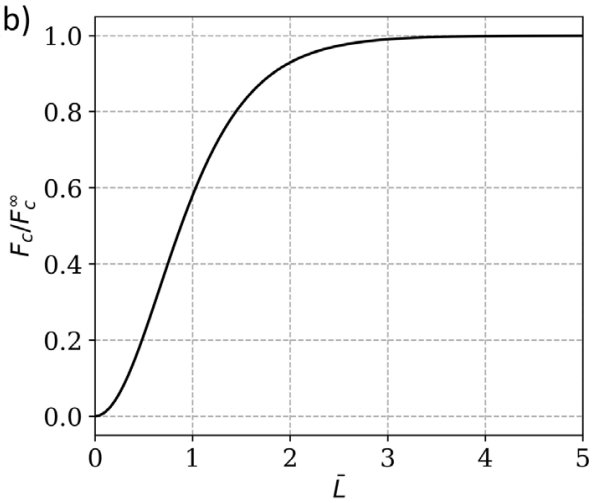

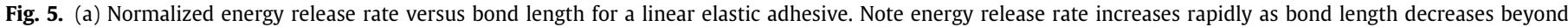

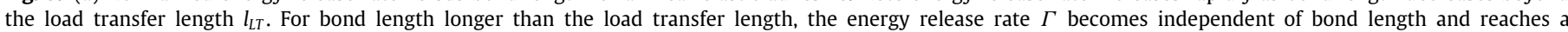

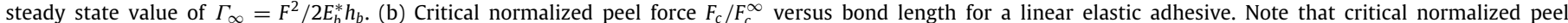
force goes to zero as bond length become small. Fig. $5 \mathrm{~b}$ will be discussed in the following section.

For this case, the critical peel force $F_{c}^{\infty}$ needed to initiate crack growth is given by

$F_{c}^{\infty}=\sqrt{2 E_{b}^{*} h_{b} G_{c}}$.

The superscript $\infty$ indicates that this result is valid for long bond lengths. Eq. (24a) suggests it is advantageous to have stiff and thick backing layer as it increases the peel force without changing the adhesion. In other words, one can increase the peel force of a weak interface by geometry. This conclusion has been justified by experiments $[19,26,42,43]$. These experiments showed that the critical force is proportional to $1 / \sqrt{C}$ where $C$ is the compliance of the system, and $C \sim 1 / E_{b}^{*} h_{b}$ for the zero degree peel test [19]. Note that the only place where the adhesive properties appear is the critical fracture energy $G_{c}$.

The critical peel force $F_{c}$ drops dramatically as the bond length decreases (see Fig. 5(b)). Indeed, Eq. (18) implies that the fracture condition (22) is

$F_{c}=F_{c}^{\infty} \tanh ^{2}\left(L / l_{L T}\right)$.

Since $\tanh ^{2}\left(L / l_{L T}\right) \approx\left(L / l_{L T}\right)^{2}$ for small $L / l_{L T}$, the critical peel force to grow the crack decreases rapidly with decreasing bond length.

(3) A short bond length destabilizes crack growth. Note that the first term of Eq. (20), $F^{2} / 2 E_{b}^{*} h_{b}$, does not change with bond length or adhesive properties whereas the second term does. In our system, extension of the interface crack by $\Delta c>0$ is exactly $-\Delta L$, that is, the bond length decreases by the amount of crack extension. Thus, the second term in Eq. (20) controls the change of energy release rate as the crack extends. Note this term increases with increasing crack length (or decreasing $L$ ). If the interfacial fracture energy is a material constant, then crack growth is stable if

$$
\left.\frac{\partial \Gamma}{\partial c}\right|_{F}=-\left.\frac{\partial \Gamma}{\partial L}\right|_{F}<0
$$

In our case $\left.\frac{\partial \Gamma}{\partial c}\right|_{F}>0$, so the condition equation (25) can never be satisfied as long as the critical energy is a constant independent of the amount of crack extension; the best one can manage is neutral stability $\left.\frac{\partial \Gamma}{\partial c}\right|_{F}=0$, which occurs at long bond lengths, i.e., $L / l_{L T} \gg 1$.

In Fig. 6, we plot the normalized energy release rate

$\frac{\Gamma}{\Gamma_{\infty}}=1+\frac{\Gamma_{L}}{\Gamma_{\infty}}$

versus $\bar{L}$ for four different values of normalized peel force $\bar{F}$ (dashed lines). We also plotted the prediction of the linear theory by Eq. (18) in the same figure (solid line). For linear theory,

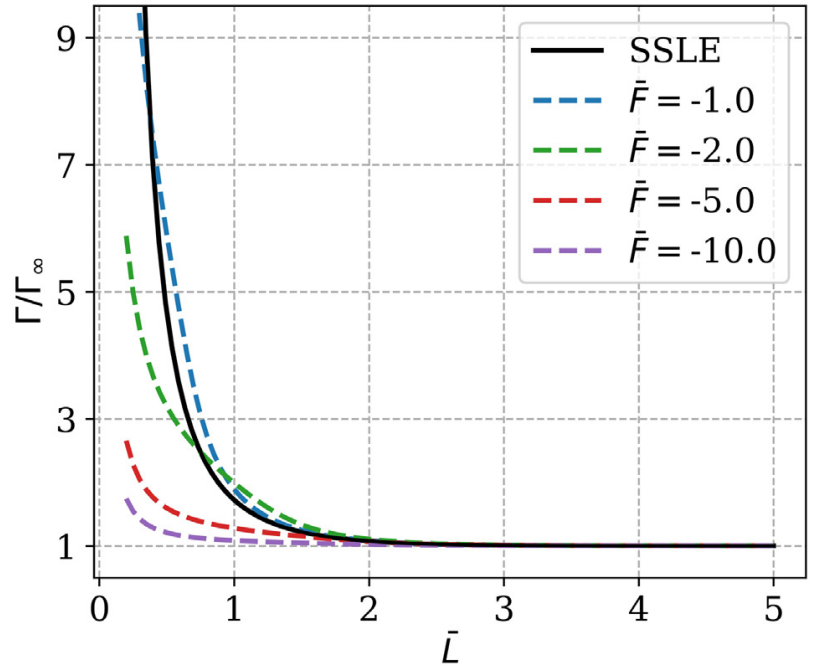

Fig. 6. Normalized energy release rate $\Gamma / \Gamma_{\infty}$ versus normalized bond length $\bar{L}$ for different normalized peel force $\bar{F}$. The predictions of SSLE and NL are plotted as the solid line and dashed lines, respectively. Note normalized energy release rate is independent of the peel force in the linear theory.

$\Gamma / \Gamma_{\infty}$ is independent of peel force - that is, the energy release rate scales as the square of the peel force. Surprisingly, the normalized energy release rate $\Gamma / \Gamma_{\infty}$ predicted by the nonlinear theory at $a$ given bond length decreases with the peel force: the linear theory dramatically overestimates the energy release rate at large peel force. A striking example is to consider large peel force (e.g. $\bar{F}=$ -10 ); according to Fig. 6 (purple dashed line), $\Gamma$ is approximately independent of the bond length for $\bar{L} \geq 1 / 2$ and is given by $\Gamma \approx \Gamma_{\infty}=F^{2} / 2 E_{b}^{*} h_{b}$. However, the energy release rate based on the linear theory (see Eq. (18)) predicts that energy release rate increases rapidly with decreasing bond length for $1 / 2 \leq$ $\bar{L} \leq 2$. This surprising result is due to the fact that the linear theory overestimates $\gamma_{\min }$ in this regime (see Fig. 2) and hence overestimates the energy release rate (see Eq. (20)). Finally, it is necessary to point out that the shear-lag model may not be accurate at $x_{1}=L$ because of a stress concentration at the free edge (due to edge effects). Thus, the results in this section should be checked against FE result in the next section. 


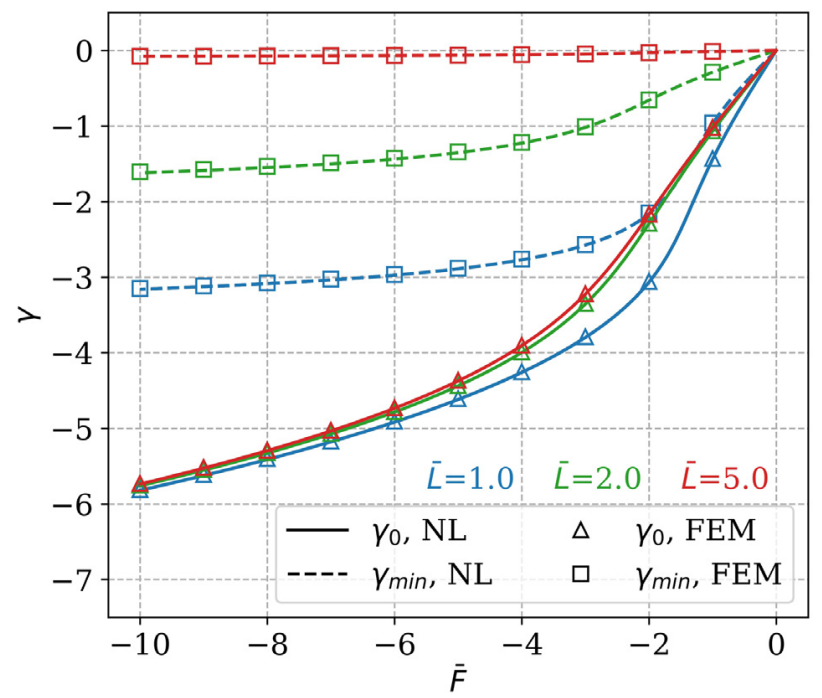

Fig. 7. Maximum shear strain $\gamma_{0}$ and minimum shear strain $\gamma_{\text {min }}$ versus normalized peel force $\bar{F}$ for different normalized bond length $\bar{L}$ (blue, green and red colors indicate $\bar{L}=1.0,2.0$ and 5.0). $\gamma_{0}$ and $\gamma_{\text {min }}$ of nonlinear shear-lag model are plotted as solid and dashed lines respectively. FEM results are represented as symbols.

\subsection{Stresses in the adhesive layer}

As shown in our previous work [38], an important distinction between the linear and nonlinear theory is that the stress state in the adhesive layer is entirely different. In the linear theory, the stress state is one dimensional, that is, the only non-vanishing stress component is the shear stress. However, under large deformation, the stress state in the adhesive is three dimensional. Indeed, the true normal stress $\tau_{11}$ in the adhesive layer is not zero and is in fact much greater than $\tau_{12}$ for large peel force. The inplane stresses in the adhesive layer are completely determined by the shear strain $\gamma$ distribution and the hardening function $f$,

$\tau_{11}=\mu \gamma^{2} f(\gamma), \tau_{12}=\mu \gamma f(\gamma)$, and $\tau_{22} \approx 0$.

Note for small shear strains (small applied force), the transverse stress $\tau_{11}$ is insignificant compare to the shear stress, consistent with the linear theory, where it is assumed to be zero.

\section{Finite element analysis}

\subsection{Finite element method (FEM) results}

In the following, we use a nonlinear FE model to check the results of the shear-lag model in the previous section and to study edge effects. These FE calculations account for nonlinear material behavior using the three term Yeoh's material behavior and large strain kinematics. All simulations are carried out using ABAQUS and plane strain deformation is assumed (as in the shear-lag model). Since the FE model is similar to our previous work, relevant details are given in the SI.

Fig. 7 plots the maximum shear strain $\gamma_{0}$ and minimum shear strain $\gamma_{\text {min }}$ in the adhesive layer versus normalized peel force $\bar{F}$ for different normalized bond length $\bar{L}$. The blue, green and red colors indicate $\bar{L}=1.0,2.0$ and 5.0. The nonlinear shearlag solution of $\gamma_{0}$ and $\gamma_{\min }$ are plotted as solid and dashed lines respectively. FEM results are represented as symbols for comparison. Fig. 7 shows excellent agreement between predictions of nonlinear shear-model and FEM results.

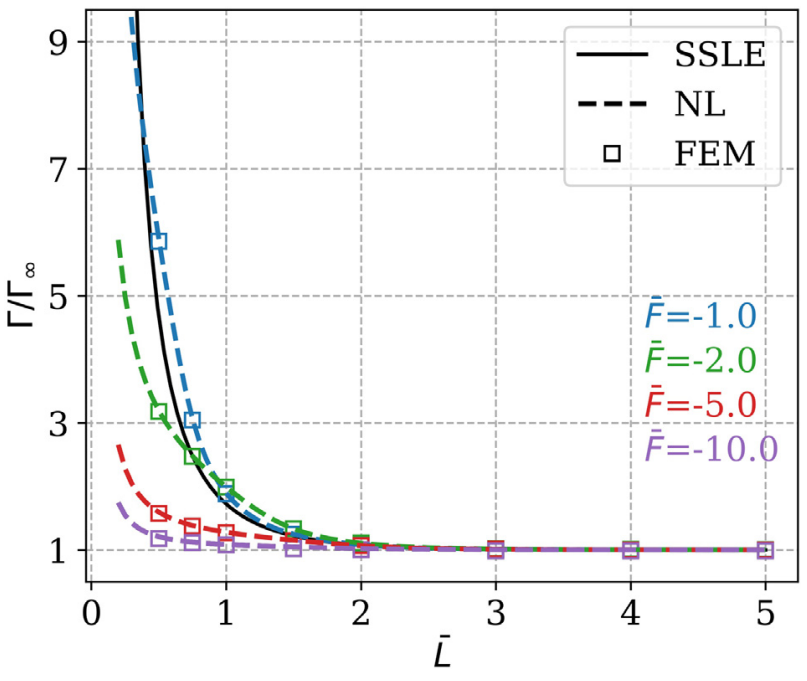

Fig. 8. Normalized energy release rate $\Gamma / \Gamma_{\infty}$ versus normalized bond length $\bar{L}$ for different normalized peel force $\bar{F}$. The blue, green, red and purple colors correspond to $\bar{F}=-1.0,-2.0,-5.0$ and -10.0 . The FEM results are represented by symbols. SSLE and NL solution are plotted as the solid line and dashed lines, respectively.

\subsection{Energy release rate and stress distribution in adhesive layer}

ABAQUS can also evaluate the energy release rate and these energy release rates (symbols) are compared with the energy release rates obtained using the nonlinear shear-lag model given by Eq. (20) (dashed lines) in Fig. 8. The agreement between our analytic model and FEM is excellent. This justifies our results in Section 2, in particular, Eq. (20).

Fig. 9 compares the true stress distributions $\tau_{12}, \tau_{11}$ and $\tau_{22}$ in the adhesive layer obtained by our FEM with our nonlinear model (27) for $\bar{F}=-10$. All stresses are normalized by the maximum stress. The normalized position $\bar{r}$ is defined by $\bar{r} \equiv x_{1} / L$. The nonlinear shear-lag model is able to capture accurately the true stress distribution accurately as long as (27) is used to describe the stress state of the adhesive layer and the edges at $x_{1}=0, L$ are avoided. In the next section, we focus on the stress state near these edges, where the shear-lag model is expected to break down.

\subsection{Edge effects}

The FEM results in Fig. 7 to 9 show that the nonlinear model accurately captures the stress and strain in the adhesive layer as long as one avoids the edges at $x_{1}=0$ and $L$. This is expected, since the edge at $x_{1}=0$ is the tip of an interface crack and the other a sharp $90^{\circ}$ corner. Since one of the key assumptions of the shear-lag model is that the stress and strain is independent of the vertical coordinate, this model cannot possibly capture the true state of stress at these locations. Indeed, the stress distributions can be significantly different along the adhesive/substrate and adhesive/backing interfaces near these edges. In the following, we use our FEM model to study these edge stress states. All figures below use the coordinates in the deformed configuration. Specifically, the true stresses are plotted against the deformed coordinates $y_{\alpha}$. An illustration of the difference between the reference coordinate (undeformed configuration, where a material point is denoted by its position $x_{\alpha}$ ) and deformed coordinates (where the material point $x_{\alpha}$ moves to $y_{\alpha}$ ) is shown in the bottom of Fig. 11(a). Note, on the adhesive/substrate interface, there is no difference between deformed and undeformed coordinates, 

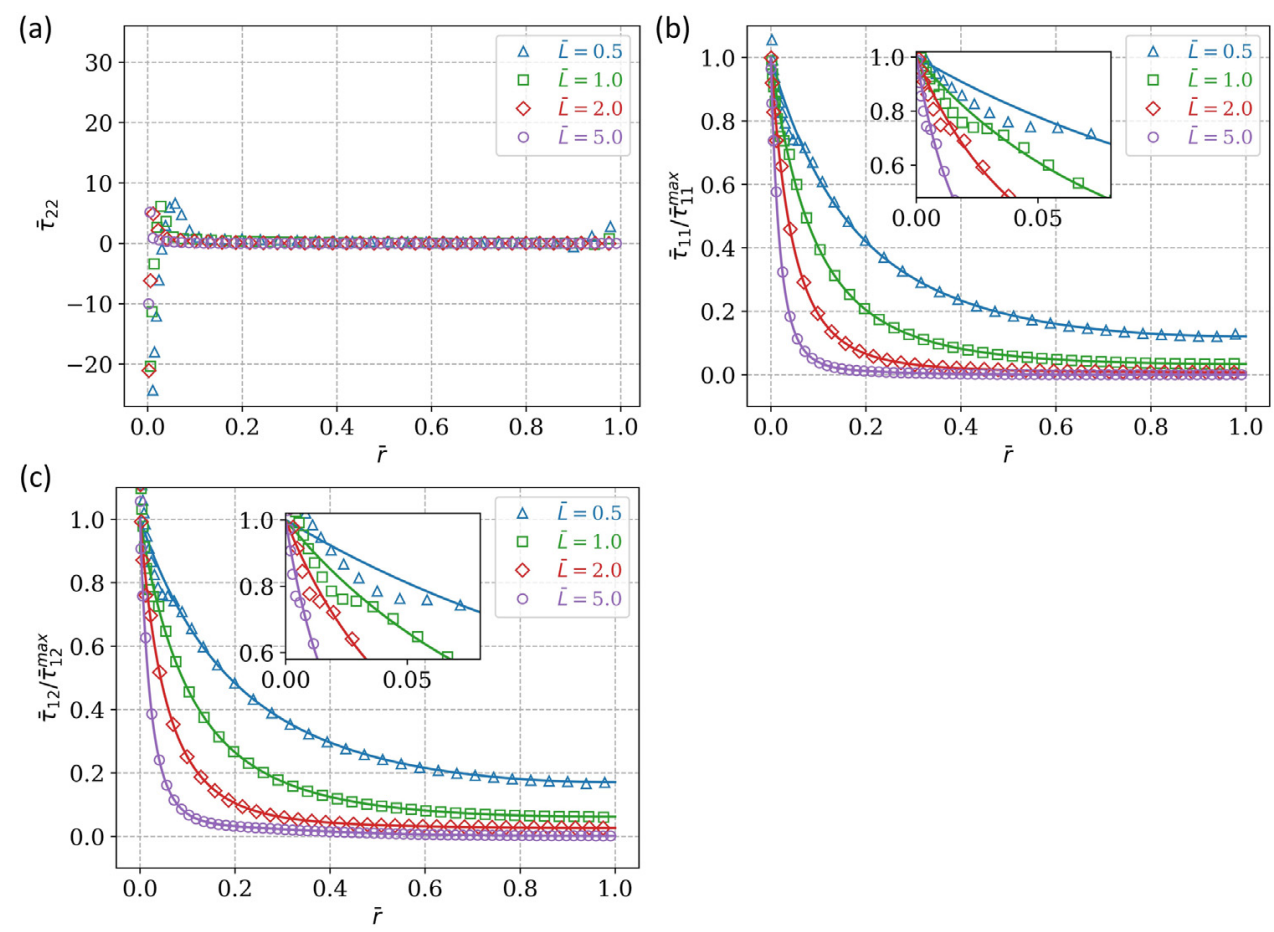

Fig. 9. Comparison of true stress distributions in the adhesive layer obtained using nonlinear shear-lag model (solid lines) and finite element models (symbols) for four different bond lengths. The normalized applied force is $\bar{F}=-10$. (a) $\bar{\tau}_{22}$; (b) $\bar{\tau}_{11} / \bar{\tau}_{11}^{\max }$; and (c) $\bar{\tau}_{12} / \bar{\tau}_{12}^{\max }$. The maximum stress $\bar{\tau}_{11}^{\max }$ for different bond lengths $\bar{L}=0.5,1.0,2.0,5.0$ are $351,312,292$ and 285 , respectively; and the maximum stress $\bar{\tau}_{12}^{\max }$ for these different bond lengths are $-59,-53,-50$ and -49 , respectively.
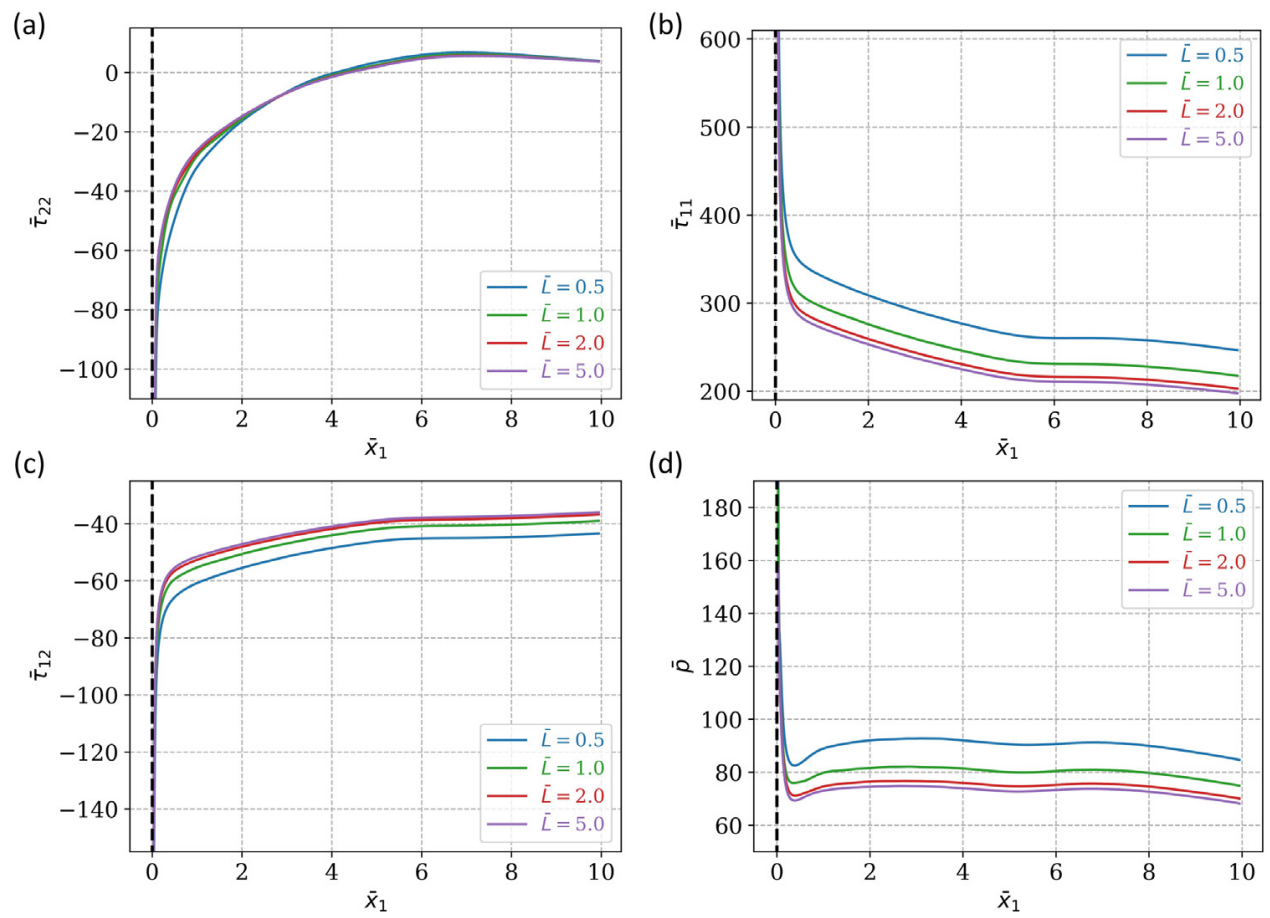

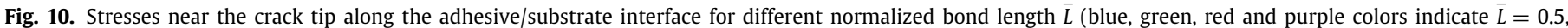

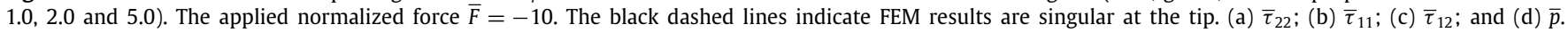

i.e., $x_{\alpha}=y_{\alpha}$, as the substrate is rigid and we assume perfect bonding.

Figs. 10 and 11 plot the normalized stress components along the adhesive/substrate and adhesive/backing interfaces near the crack tip respectively. Here we used different normalized coordinates to reflect our focus near the crack tip. The coordinates in the reference and deformed configurations are normalized by the initial adhesive thickness $h$ and are denoted by $\bar{x}_{\alpha}=x_{\alpha} / h$ and $\bar{y}_{\alpha}=y_{\alpha} / h$ respectively. Fig. 10(a) shows that there is a very large stress concentration directly ahead of the interfacial crack tip. Note that the normalized stress $\bar{\tau}_{22}$ is very large and compressive. However, the hydrostatic stress $\bar{p} \equiv\left(\bar{\tau}_{11}+\bar{\tau}_{22}+\bar{\tau}_{33}\right) / 3$ is still 


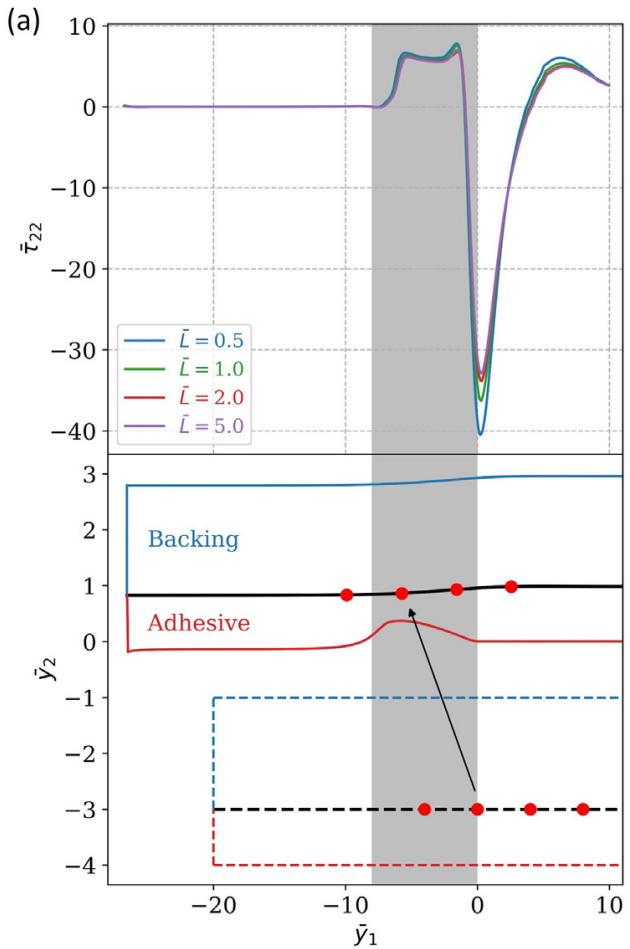

(c)
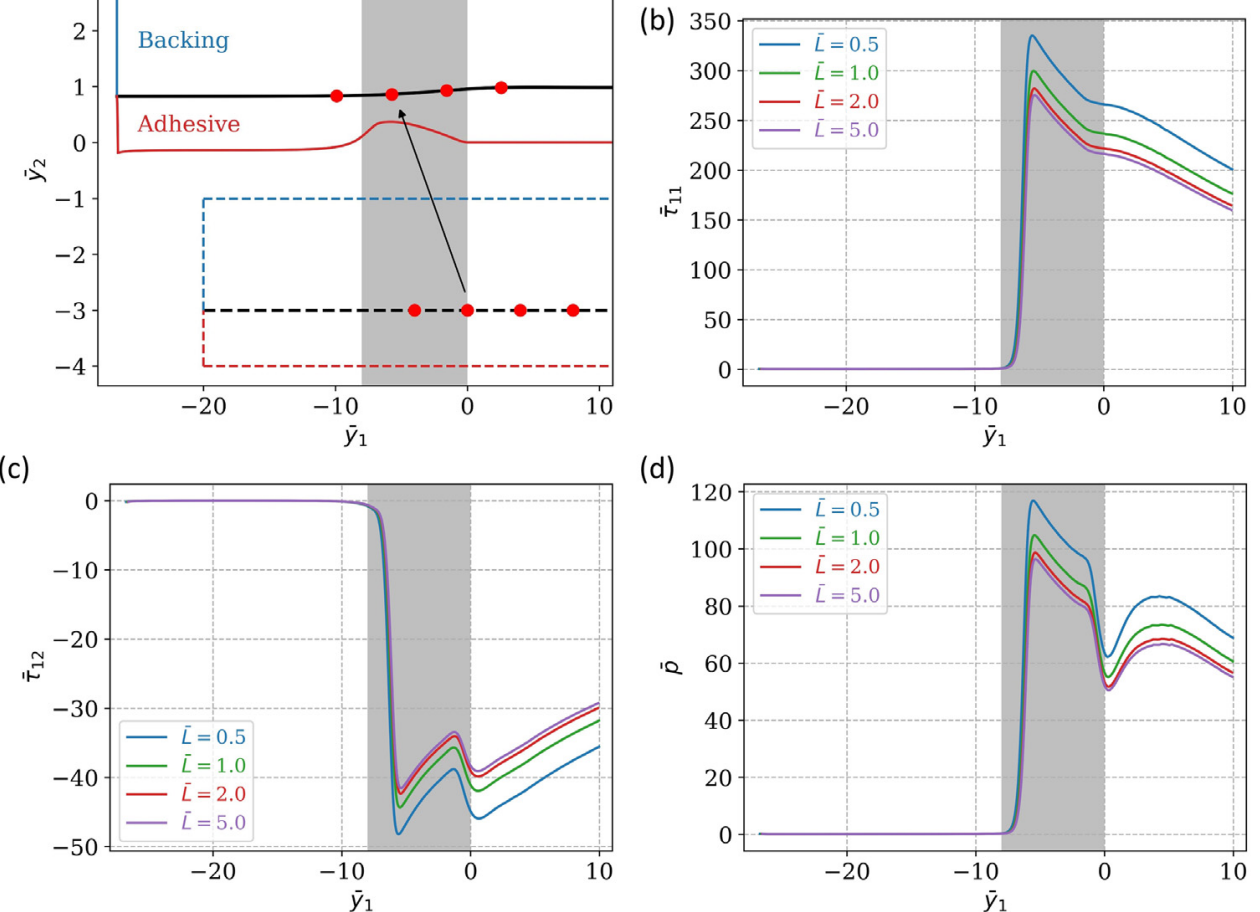

(d)

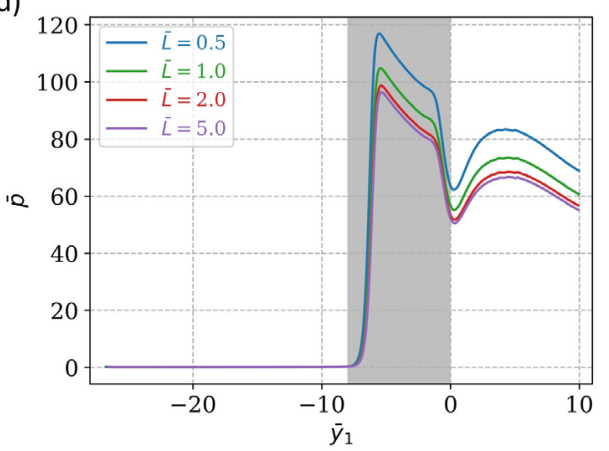

Fig. 11. Stresses near the crack tip along the adhesive/backing interface for different normalized bond length $\bar{L}$ (blue, green, red and purple colors indicate $\bar{L}=0.5$, 1.0, 2.0 and 5.0). The applied normalized force is $\bar{F}=-10$. (a) $\bar{\tau}_{22}$; (b) $\bar{\tau}_{11}$; (c) $\bar{\tau}_{12}$; and (d) $\bar{p}$.

very high and tensile near the crack tip, this is due to the large normal stress parallel to the interface. It must be noted that here we assume the interface is perfectly bonded whereas in reality, local slip or debonding will relax these extremely high stresses near the crack tip. As expected, the stress concentration is much less severe along the adhesive/backing interface. Note, however, the hydrostatic stress here is still very large and tensile.

Figs. 12 and 13 plot the normalized stress components along the adhesive/substrate and adhesive/backing interfaces near the right edge corner at $x_{1}=L$ respectively. As shown in the bottom of Fig. 13, we define the distances measuring from the right corner along the adhesive/substrate and adhesive/backing interfaces by $m_{1}$ and $n_{1}$, and these distances are normalized by the adhesive thickness $h$, i.e., $\bar{m}_{1}=m_{1} / h$ and $\bar{n}_{1}=n_{1} / h$. These figures show that the stresses on both interfaces increase rapidly as the bond length decreases beyond one load transfer length. Further, the stress state at this edge along the adhesive-back interface is triaxial with $\tau_{22}, \tau_{11}, \tau_{33}$ all positive; therefore, cavitation is likely to occur at this edge. However, for bond length longer than 2 times the load transfer length, these stresses are small, and debonding or cavitation here is less likely.

\section{Summary and discussion}

In zero degree peel, the dominant deformation in the adhesive layer is shear. Except at distances very close to the peel front and the free edge, finite element analysis has shown that the shear strain and the stresses in the adhesive layer is well predicted by our analytic model in Section 2 . Here we recall that in large deformation, even simple shear can induce normal stresses via the Poynting effect [44]. Thus, even though the dominant deformation mode in the adhesive layer is shear, the stress state there is not one dimensional. Indeed, at large shear strains, the adhesive is subjected to a normal stress $\tau_{11}$ (in the direction of the peel force) that is much larger than the shear stress $\tau_{12}$, as noted by (27). This, together with the out of plane stress due to the plane strain confinement, raise the possibility of cavitation inside the adhesive layer despite the fact that the stress normal to the adhesive layer $\tau_{22}$ is zero.

Due to strain hardening, the minimum shear strain $\gamma_{\min }$ at the free end of the tape depends primarily on the bond length and is quite insensitive to the peel force, as long as the peel force is large. This result significantly affects the energy release rate $\Gamma$, which consists of two parts: the first is the energy release rate of an infinite long tape, $\Gamma_{\infty}$ which is independent of the mechanical 

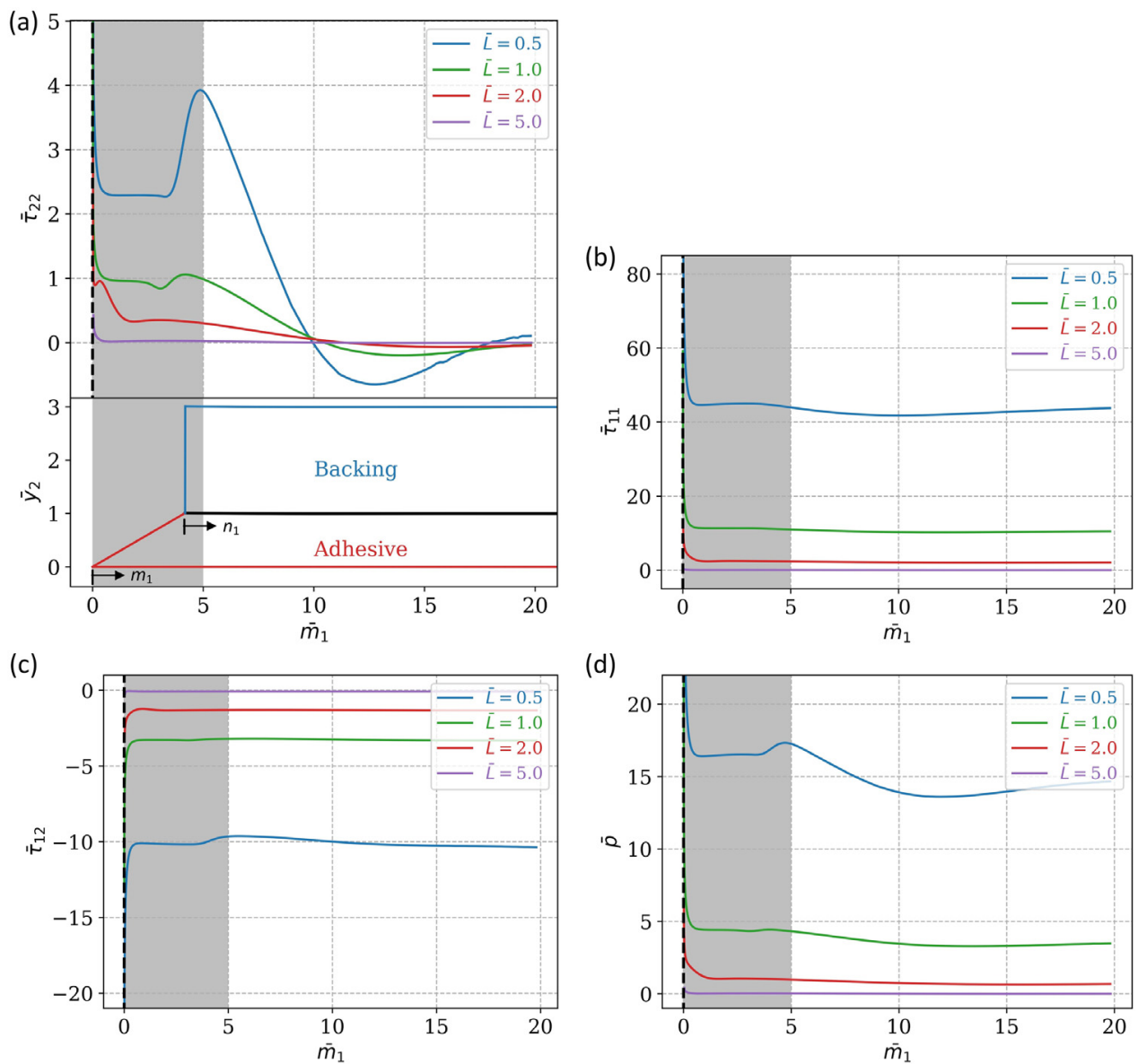

(d)

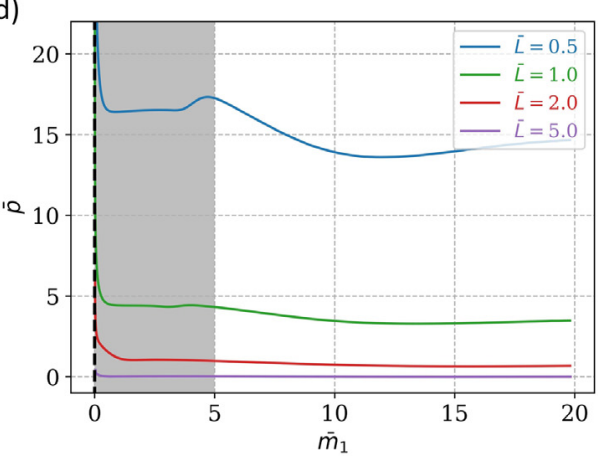

Fig. 12. Stresses near the corner along the adhesive/substrate interface for different normalized bond length $\bar{L}$ (blue, green red and purple colors indicate $\bar{L}=0.5$, 1.0, 2.0 and 5.0). The applied normalized force $\bar{F}=-10$. The black dashed lines indicate FEM results are singular at the corner. (a) $\bar{\tau}_{22}$; (b) $\bar{\tau}_{11}$; (c) $\bar{\tau}_{12}$; and (d) $\bar{p}$.
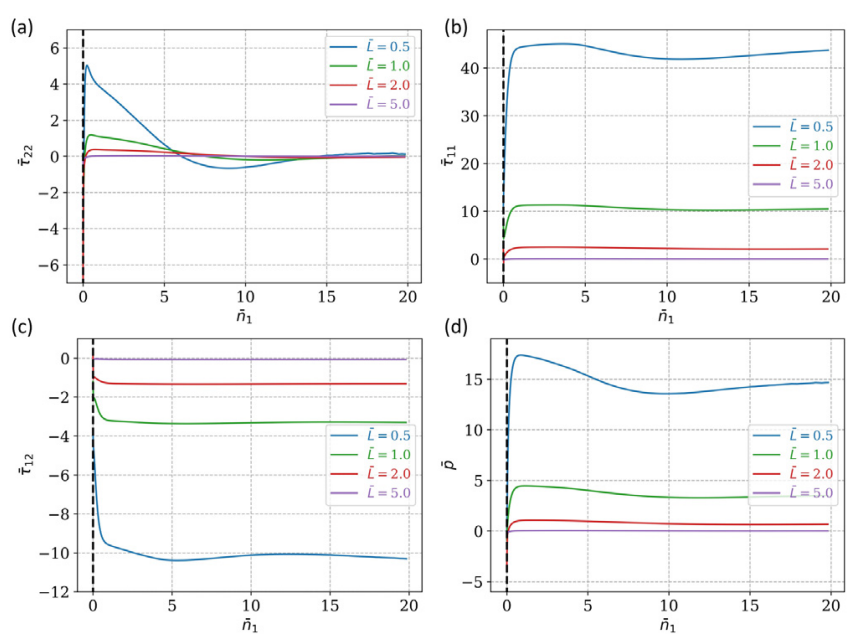

Fig. 13. Stresses near the corner along the adhesive/backing interface for different normalized bond length $\bar{L}$ (blue, green red and purple colors indicate $\bar{L}=0.5,1.0,2.0$ and 5.0). The applied normalized force $\bar{F}=-10$. The black dashed lines indicate FEM results are singular at $\bar{s}=0$ (1) $\bar{\tau}_{22}$; (2) $\bar{\tau}_{11}$; (3) $\bar{\tau}_{12}$; and (4) $\bar{p}$.

property of the adhesive and the second part depends on $\gamma_{\text {min }}$ which is insensitive to the peel force in the nonlinear high force regime. As a result, $\Gamma / \Gamma_{\infty}$ increases much slower with decreasing bond length, which means that the driving force for unstable crack growth is reduced significantly in comparison with linear theory. It is interesting to note that for long tapes (i.e., $L \gg l_{L T}$ ) $\gamma_{\text {min }}$ is practically zero, this means that the force to drive the crack is controlled by the stiffness of backing layer and is independent of the mechanical behavior of adhesive. For a stiff backing, the force to pull the tape off can be very high. Thus, even though the force is supported by a small segment of the tape, the part of the tape that is not supporting shear actually plays an important role in protecting the tape as it significantly reduces the energy release rate. This is one reason to use a longer tape in the hanging weight experiment.

The maximum shear strain is found to increase much less rapidly with the applied force in the nonlinear model. Furthermore, in the high force regime it is insensitive to the bond length. However, it should be aware that the maximum shear strain evaluated using the shear-lag model is most likely a lower bound because of the high stress concentration and 3D effect near the peel front. Here, our FE results indicate the normal stress $\tau_{22}$ on the adhesive/substrate interface close to the peel front is highly compressive, so cavitation, if exist, is expected to occur at a small distance away from the peel front, where the normal stress is tensile. In contrast, the normal stress $\tau_{22}$ on both the adhesive/substrate and the adhesive/backing interfaces close to the free edge are found to be tensile. For short bond lengths, the stress state on the interfaces near the free edge can be very high, and cavitation here is a definite possibility.

A limitation of our model is that the adhesive is assumed to be elastic while in reality they are nonlinear viscoelastic. The 
main difficulty with extending our analysis to account for viscoelastic effect is the absence of an accurate 3D large deformation viscoelastic model for adhesives.

Finally, although this study is motivated by understanding how adhesive tapes work under predominantly shear loading, our results have much wider generality and can be applied to study the mechanics of other composite systems with similar geometry. For example, the energy release rate expression and the stresses in the adhesive layer as well as the solution of the nonlinear shear-lag model, are valid for a wide class of hyperelastic strain energy density functions $W$, as shown in the supplementary information.

\section{Acknowledgments}

C.Y. Hui acknowledges National Science Foundation (NSF), USA MoMS program under grant number 1903308. H. K. Minsky is supported by the $3 M$ Company, USA and Centre national de la recherché scientifique, France. We are grateful to the reviewers for their helpful comments.

\section{Appendix A. Supplementary data}

Supplementary material related to this article can be found online at https://doi.org/10.1016/j.eml.2019.100518.

\section{References}

[1] Y. Wei, J.W. Hutchinson, Interface strength, work of adhesion and plasticity in the peel test, in: W.G. Knauss, R.A. Schapery (Eds.), Recent Advances in Fracture Mechanics: Honoring Mel and Max Williams, Springer Netherlands, Dordrecht, 1998, pp. 315-333, http://dx.doi.org/10.1007/978-94017-2854-6_16.

[2] D.H. Kaelble, Theory and analysis of peel adhesion: Mechanisms and mechanics, Trans. Soc. Rheol. 3 (1959) 161-180, http://dx.doi.org/10.1122/ 1.548850 .

[3] K. Kendall, Thin-film peeling-the elastic term, J. Phys. D: Appl. Phys. 8 (1975) 1449-1452, http://dx.doi.org/10.1088/0022-3727/8/13/005.

[4] C. Creton, M. Ciccotti, Fracture and adhesion of soft materials: a review, Rep. Progr. Phys. 79 (2016) 046601, http://dx.doi.org/10.1088/0034-4885/ 79/4/046601.

[5] R. Villey, P.-P. Cortet, C. Creton, M. Ciccotti, In-situ measurement of the large strain response of the fibrillar debonding region during the steady peeling of pressure sensitive adhesives, Int. J. Fract. 204 (2017) 175-190, http://dx.doi.org/10.1007/s10704-016-0171-1.

[6] D.H. Kaelble, Peel adhesion: Micro-fracture mechanics of interfacial unbonding of polymers, Trans. Soc. Rheol. 9 (1965) 135-163, http://dx.doi. org/10.1122/1.549022.

[7] C. Creton, H. Lakrout, Micromechanics of flat-probe adhesion tests of soft viscoelastic polymer films, J. Polym. Sci. Polym. Phys. 38 (2000) 965-979, http://dx.doi.org/10.1002/(SICI)1099-0488(20000401)38:7<965:: AID-POLB7>3.0.CO;2-8.

[8] H. Lakrout, C. Creton, D. Ahn, K.R. Shull, Influence of molecular features on the tackiness of acrylic polymer melts, Macromolecules 34 (2001) 7448-7458, http://dx.doi.org/10.1021/ma0020279.

[9] A. Zosel, The effect of fibrilation on the tack of pressure sensitive adhesives, Int. J. Adhes. Adhes. 18 (1998) 265-271, http://dx.doi.org/10.1016/S01437496(98)80060-2.

[10] H. Lakrout, P. Sergot, C. Creton, Direct observation of Cavitation and fibrillation in a probe tack experiment on model acrylic pressuresensitive-adhesives, J. Adhes. 69 (1999) 307-359, http://dx.doi.org/10.1080/ 00218469908017233.

[11] A.J. Crosby, K.R. Shull, H. Lakrout, C. Creton, Deformation and failure modes of adhesively bonded elastic layers, J. Appl. Phys. 88 (2000) 2956-2966, http://dx.doi.org/10.1063/1.1288017.

[12] K.R. Shull, D. Ahn, W.-L. Chen, C.M. Flanigan, A.J. Crosby, Axisymmetric adhesion tests of soft materials, Macromol. Chem. Phys. 199 (1998) 489-511, http://dx.doi.org/10.1002/(SICI)1521-3935(19980401)199: 4<489::AID-MACP489>3.0.CO;2-A.

[13] Y.Y. Lin, C.-Y. Hui, H.D. Conway, A detailed elastic analysis of the flat punch (tack) test for pressure-sensitive adhesives, J. Polym. Sci. Polym. Phys. 38 (2000) 2769-2784, http://dx.doi.org/10.1002/1099-0488(20001101)38: 21<2769::AID-POLB60>3.0.CO;2-J.
[14] A.N. Gent, C. Wang, Fracture mechanics and cavitation in rubber-like solids, J. Mater. Sci. 26 (1991) 3392-3395, http://dx.doi.org/10.1007/BF01124691.

[15] D.H. Kaelble, Theory and analysis of peel adhesion: Bond stresses and distributions, Trans. Soc. Rheol. 4 (1960) 45-73, http://dx.doi.org/10.1122/ 1.548868 .

[16] A.N. Gent, Adhesion of viscoelastic materials to rigid substrates. II. Tensile strength of adhesive joints, J. Polym. Sci. Polym. Phys. 9 (1971) 283-294, http://dx.doi.org/10.1002/pol.1971.160090205.

[17] A.N. Gent, R.P. Petrich, Adhesion of viscoelastic materials to rigid substrates, Proc. R. Soc. Lond. Ser. A Math. Phys. Eng. Sci. 310 (1969) 433-448, http://dx.doi.org/10.1098/rspa.1969.0085.

[18] A. Zosel, Shear strength of pressure sensitive adhesives and its correlation to mechanical properties, J. Adhes. 44 (1994) 1-16, http://dx.doi.org/10. 1080/00218469408026613.

[19] S. Ponce, J. Bico, B. Roman, Effect of friction on the peeling test at zero-degrees, Soft Matter 11 (2015) 9281-9290, http://dx.doi.org/10.1039/ C5SM01203A.

[20] A.J. Kinloch, Adhesion and Adhesives: Science and Technology, Springer Science \& Business Media, 2012

[21] R.R. Collino, N.R. Philips, M.N. Rossol, R.M. McMeeking, M.R. Begley, Detachment of compliant films adhered to stiff substrates via van der Waals interactions: role of frictional sliding during peeling, J. R. Soc. Interface 11 (2014) 20140453, http://dx.doi.org/10.1098/rsif.2014.0453.

[22] A.R. Mojdehi, D.P. Holmes, D.A. Dillard, Friction of extensible strips: An extended shear lag model with experimental evaluation, Int. J. Solids Struct. 124 (2017) 125-134, http://dx.doi.org/10.1016/j.ijsolstr.2017.06.021.

[23] F. Sosson, A. Chateauminois, C. Creton, Investigation of shear failure mechanisms of pressure-sensitive adhesives, J. Polym. Sci. Polym. Phys. 43 (2005) 3316-3330, http://dx.doi.org/10.1002/polb.20619.

[24] S.M. Xia, L. Ponson, G. Ravichandran, K. Bhattacharya, Adhesion of heterogeneous thin films-I: Elastic heterogeneity, J. Mech. Phys. Solids 61 (2013) 838-851, http://dx.doi.org/10.1016/j.jmps.2012.10.014.

[25] T. Cohen, C.U. Chan, L. Mahadevan, Competing failure modes in finite adhesive pads, Soft Matter 14 (2018) 1771-1779, http://dx.doi.org/10.1039/ C7SM02378B.

[26] M.D. Bartlett, A.B. Croll, A.J. Crosby, Designing bio-inspired adhesives for shear loading: From simple structures to complex patterns, Adv. Funct. Mater. 22 (2012) 4985-4992, http://dx.doi.org/10.1002/adfm.201201344.

[27] K. Kendall, The adhesion and surface energy of elastic solids, J. Phys. D: Appl. Phys. 4 (1971) 1186-1195, http://dx.doi.org/10.1088/0022-3727/4/8/ 320.

[28] M.D. Thouless, Q.D. Yang, A parametric study of the peel test, Int. J. Adhes. Adhes. 28 (2008) 176-184, http://dx.doi.org/10.1016/j.ijadhadh.2007.06. 006.

[29] Q.D. Yang, M.D. Thouless, Mixed-mode fracture analyses of plasticallydeforming adhesive joints, Int. J. Fract. 110 (2001) 175-187, http://dx.doi. org/10.1023/A:1010869706996.

[30] Z. Suo, J.W. Hutchinson, Interface crack between two elastic layers, Int. J. Fract. 43 (1990) 1-18, http://dx.doi.org/10.1007/BF00018123.

[31] J. Chopin, R. Villey, D. Yarusso, E. Barthel, C. Creton, M. Ciccotti, Nonlinear viscoelastic modeling of adhesive failure for polyacrylate pressure-sensitive adhesives, Macromolecules 51 (2018) 8605-8610, http://dx.doi.org/10. 1021/acs.macromol.8b01374.

[32] A. Bellamine, E. Degrandi, M. Gerst, R. Stark, C. Beyers, C. Creton, Design of nanostructured waterborne adhesives with improved shear resistance, Macromol. Mater. Eng. 296 (2011) 31-41, http://dx.doi.org/10.1002/mame. 201000265.

[33] A. Lindner, B. Lestriez, S. Mariot, C. Creton, T. Maevis, B. Lühmann, R. Brummer, Adhesive and rheological properties of lightly crosslinked model acrylic networks, J. Adhes. 82 (2006) 267-310, http://dx.doi.org/10.1080/ 00218460600646594.

[34] F. Tanguy, M. Nicoli, A. Lindner, C. Creton, Quantitative analysis of the debonding structure of soft adhesives, Eur. Phys. J. E 37 (2014) 3, http: //dx.doi.org/10.1140/epje/i2014-14003-8.

[35] M.C. Boyce, E.M. Arruda, Constitutive models of rubber elasticity: A review, Rubber Chem. Technol. 73 (2000) 504-523, http://dx.doi.org/10.5254/1. 3547602.

[36] K. Kendall, Crack propagation in lap shear joints, J. Phys. D: Appl. Phys. 8 (1975) 512-522, http://dx.doi.org/10.1088/0022-3727/8/5/010.

[37] M.S. Kafkalidis, M.D. Thouless, The effects of geometry and material properties on the fracture of single lap-shear joints, Int. J. Solids Struct. 39 (2002) 4367-4383, http://dx.doi.org/10.1016/S0020-7683(02)00344-X.

[38] C.-Y. Hui, Z. Liu, H. Minsky, C. Creton, M. Ciccotti, Mechanics of an adhesive tape in a zero degree peel test: effect of large deformation and material nonlinearity, Soft Matter 14 (2018) 9681-9692, http://dx.doi.org/10.1039/ C8SM01731J.

[39] D.H. Kaelble, Theory and analysis of peel adhesion: Adhesive thickness effects, J. Adhes. 37 (1992) 205-214, http://dx.doi.org/10.1080/ 00218469208031262 
[40] J.R. Rice, A path independent integral and the approximate analysis of strain concentration by notches and cracks, J. Appl. Mech. 35 (1968) 379-386, http://dx.doi.org/10.1115/1.3601206.

[41] B. Budiansky, J.R. Rice, Conservation laws and energy-release rates, J. Appl. Mech. 40 (1973) 201, http://dx.doi.org/10.1115/1.3422926.

[42] D.R. King, A.J. Crosby, Optimizing adhesive design by understanding compliance, ACS Appl. Mater. Interfaces 7 (2015) 27771-27781, http://dx.doi. org/10.1021/acsami.5b08934.
[43] M.D. Bartlett, A.J. Crosby, High capacity easy release adhesives from renewable materials, Adv. Mater. 26 (2014) 3405-3409, http://dx.doi.org/ 10.1002/adma.201305593.

[44] J.H. Poynting, On pressure perpendicular to the shear planes in finite pure shears, and on the lengthening of loaded wires when twisted, Proc. R. Soc Lond. Ser. A Math. Phys. Eng. Sci. 82 (1909) 546-559, http://dx.doi.org/10. 1098/rspa.1909.0059. 